\title{
Estimation of Heart Rate during Exercise from a Photoplethysmographic Signal Considering Exercise Intensity
}

\author{
Takahiro Hayashi* and Tatsuya Ooi ${ }^{1}$ \\ Institute of Science and Technology, Niigata University, \\ 8050 Ikarashi-2-no-cho, Nishi-ku, Niigata-shi, Niigata, 950-2181, Japan \\ ${ }^{1}$ Graduate School of Science and Tehnoclogy, Niigata University, \\ 8050 Ikarashi-2-no-cho, Nishi-ku, Niigata-shi, Niigata, 950-2181, Japan \\ (Received October 26, 2015; accepted February 15, 2016)
}

Keywords: heart rate estimation, photoplethysmography (PPG) sensors, acceleration sensors, healthcare systems, smart sensing

Heart rate estimation during exercise with photoplethysmographic (PPG) sensors is a difficult problem because motion artifacts (MAs) contaminate PPG signals. In this paper, we propose a method of accurately estimating heart rate during exercise from a PPG signal by considering exercise intensity. The exercise intensity is estimated from acceleration signals simultaneously recorded with the PPG signal. The proposed method dynamically limits the estimation range of heart rate depending on the exercise intensity, resulting in an accurate heart rate estimation during exercise. From the experimental results, we have confirmed that the proposed method can estimate heart rate more accurately than conventional methods.

\section{Introduction}

Heart rate monitoring is important for controlling exercise intensity in rehabilitation, healthcare, and training. As a traditional method for heart rate estimation, electrocardiography (ECG) has been widely used. To monitor heart rate during exercise, chest-strap ECG devices have been developed. The cost of such devices, however, is high, and they cannot be used without expert knowledge.

Heart rate estimation from photoplethysmographic (PPG) signals has become popular recently because PPG sensors are cheaper and can be easily embedded in wearable devices like smartwatches. A PPG signal can be measured using a pulse oximeter which illuminates the skin and measures changes in light absorption. ${ }^{(1,2)}$ It is, however, difficult to accurately estimate heart rate during exercise from a PPG signal because the distance from the sensor to the skin changes when exercising, resulting in the contamination of a PPG signal by motion artifacts (MAs). ${ }^{(3)}$

There are some studies focusing on the reduction of the influence of MAs. ${ }^{(4-16)}$ Many of them, however, implicitly or explicitly assume that exercise intensity is constant or changes slightly. It is, therefore, difficult to simply apply these techniques to heart rate estimation during exercise especially when exercise intensity changes dynamically.

"Corresponding author: e-mail: hayashi@ie.niigata-u.ac.jp 
In this paper, we propose a method for accurately estimating heart rate from a PPG signal during exercise by considering the exercise intensity. To estimate the exercise intensity, the proposed method utilizes the 3D acceleration signals simultaneously recorded with a PPG signal. In this paper, we assume that a 3D acceleration sensor and a PPG sensor are integrated into a wrist-type device such as a smart watch.

The rest of the paper is organized as follows. Section 2 describes related work. Section 3 describes the proposed method. Section 4 shows the experiments we conducted for confirming the effectiveness of the proposed method. Finally, in $\$ 5$ we conclude this paper and show a future direction.

\section{Related Work}

There are some studies on reducing MAs in PPG signals. ${ }^{(4-12)}$ In these studies, the signal elements of MAs are removed by a statistical signal processing technique such as independent component analysis (ICA). Once MAs can be removed from a PPG signal, the temporal change in heart rate can be estimated by peak-frequency tracking. It is, however, difficult to completely distinguish the elements of MAs from PPG signals because statistical independence between the signals cannot be assured during exercise.

To deal with the problem of MAs, some studies have focused on the post-processing part, i.e., peak-frequency tracking. ${ }^{(13-16)}$ To avoid inaccurate estimation caused by MAs, these studies limit the estimation range of the heart rate. The methods used, however, cannot deal with rapid heart rate changes caused by changes in exercise intensity. To deal with the problem, our method accurately captures the trend of instant changes in heart rate by monitoring the change in exercise intensity with acceleration sensors.

\section{Proposed Method}

\subsection{Outline}

The proposed method estimates heart rate using a wristband-type device equipped with PPG and 3D acceleration sensors. The signals from these sensors are simultaneously recorded.

Figure 1 shows the outline of the proposed method. As the first step, the proposed method generates a spectrogram from the PPG signal using a short-time Fourier transform (STFT) [Fig. 1(a)]. At the same time, the proposed method estimates the change in exercise intensity from the 3D acceleration sensor signals [Fig. 1(b)]. Next, the proposed method performs peak-frequency tracking [Fig. 1 (c)]. In peak-frequency tracking, the spectrogram is reconstructed by emphasizing particular frequencies based on the change in exercise intensity. The details of each step are explained in the next subsections.

\subsection{Generation of spectrogram}

To grasp the temporal trend of the spectrum of a PPG signal, the proposed method generates a spectrogram. Here, let $x(t)$ be the measured PPG signal and $X(n, m)$ be the spectrogram generated by the short-time Fourier transform (STFT) with the time window size $T$. Figure 2 shows an example 


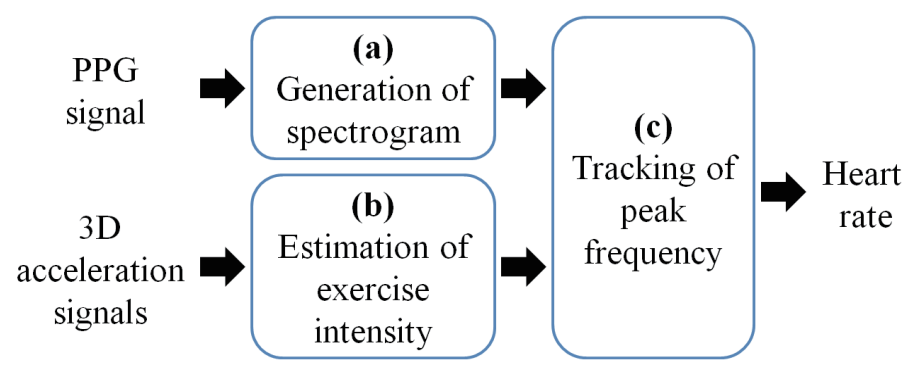

Fig. 1. (Color online) Outline of the proposed method.

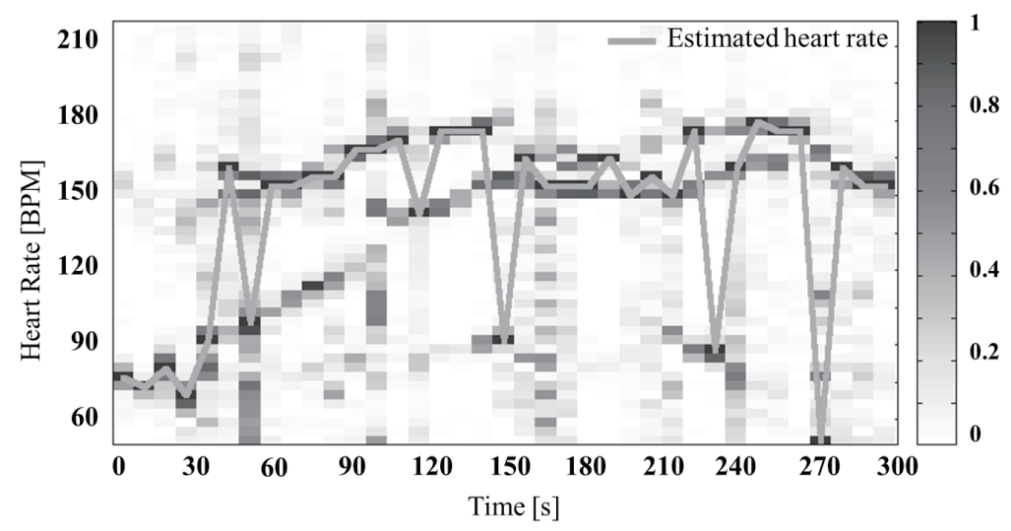

Fig. 2. Spectrogram of a PPG signal during exercise.

of a spectrogram $[T=16(\mathrm{~s})]$. In a spectrogram, the horizontal and vertical axes represent time and frequency segments, respectively. Owing to the characteristics of the STFT, the resolutions of the horizontal and vertical axes are limited to $T$ and $F[=1 / T(\mathrm{~Hz})=60 / T(\mathrm{BPM})]$, respectively. The density of color in each segment represents the power of the frequency in the segment.

The temporal change in the peak frequency of the PPG signal corresponds to heart rate if MAs are very weak. Since a PPG signal during exercise, however, is more or less contaminated by MAs, many unnatural frequency peaks appear in the spectrogram. If the technique of peak-frequency tracking is applied to such a spectrogram without any MA cancellation processing, the estimation of heart rate becomes inaccurate like the path shown in Fig. 2. It is therefore crucial to introduce a mechanism of reducing the effect of MAs. The mechanism of reducing the effect of MAs used in the proposed method is explained in $\S 3.4$.

\subsection{Estimation of exercise intensity}

Human heart rate can increase considerably within a short period of time when exercise intensity increases. On the other hand, heart rate tends to be stable when keeping exercise at a constant intensity.

The proposed method estimates the exercise intensity from the 3D acceleration sensor signals. To estimate the exercise intensity, we focus on the absolute acceleration signal $a(t)=\sqrt{a_{x}^{2}(t)+a_{y}^{2}(t)+a_{z}^{2}(t)}$, where $a_{x}(t), a_{y}(t)$, and $a_{z}(t)$ are the acceleration values on the axes. Here, let $\Delta I(n)$ be the change in exercise intensity at the time segment $n . \Delta I(n)$ is defined as 


$$
\Delta I(n)=\frac{\mu_{\mathrm{a}}(n)-\mu_{\mathrm{a}}(n-1)}{\delta_{\mathrm{a}}(n-1)} .
$$

Here, $\mu_{\mathrm{a}}(n)$ and $\sigma_{\mathrm{a}}(n)$ are, respectively, the average and $\mathrm{SD}$ of the acceleration in the $n$-th time segment defined as

$$
\mu_{\mathrm{a}}(n)=\frac{1}{T} \int_{n T}^{(n+1) T} a(t) d t
$$

and

$$
\sigma_{\mathrm{a}}(n)=\sqrt{\frac{1}{T} \int_{n T}^{(n+1) T}\left[a(t)-\mu_{\mathrm{a}}(n)\right]^{2} d t .}
$$

\subsection{Tracking of peak frequency}

To reduce the effect of MAs, the spectrogram is reconstructed by emphasizing particular frequencies as

$$
X^{\prime}(n, m)=w(n, m) X(n, m),
$$

where $w(n, m)$ is the weight matrix applied to the original spectrogram $X(n, m)$. We assume that a distribution of relative changes of heart rate can be approximated by a Gaussian distribution. In their assumption, we define the weight matrix $w(n, m)$ as

$$
w(n, m)=\frac{1}{\sqrt{2 \pi \sigma^{2} \Delta I(n)^{2}}} \exp \left\{\frac{\left[\left(m-M_{n-1}\right)-\mu \Delta I(n)\right]^{2}}{2 \sigma^{2} \Delta I(n)}\right\},
$$

where $M_{n-1}$ is the heart rate (peak frequency) of the previous time segment. The weight $w(n, m)$ dynamically changes depending on two factors: previous heart rate $M_{n-1}$ and the change in exercise intensity $\Delta I(n)$. The parameters $\mu(\Delta I)$ and $\sigma(\Delta I)$, respectively, represent the average and SD of the change in heart rate when the change in the exercise intensity is $\Delta I$.

Table 1 shows the values of $\mu(\Delta I)$ and $\sigma(\Delta I)$ used in the proposed method. These values were statistically measured using the IEEE Signal Processing Cup 2015 dataset. ${ }^{(13)}$ The dataset contains PPG signal data during exercise, acceleration sensor data, and the accurate heart rate data (ground truth data) measured by ECG. Using the heart rate and acceleration sensor data, we examined the

Table 1

Average and SD of peak frequency changes at different levels of exercise intensity change.

\begin{tabular}{lcrc}
\hline & $\Delta I$ & $\mu$ & $\sigma$ \\
\hline (i) & $\Delta I$ & 1.024 & 1.449 \\
(ii) & $\Delta I$ & 0.216 & 0.953 \\
(iii) & $\Delta I$ & -0.161 & 0.988 \\
\hline
\end{tabular}


distributions of heart rate changes along with different levels of exercise intensity change. Figure 3 shows the histograms of relative change of heart rate at different levels of $\Delta I$. By approximating the distribution shapes in these histograms as Gaussian distributions, the values in Table 1 are obtained.

To the spectrogram reconstructed by the proposed weighting [Eq. (4)], the method applies the technique of peak-frequency tracking. Figure 4 shows the original and reconstructed spectrograms. As shown in the reconstructed spectrogram, unnatural peaks can be removed. By the dynamic weighting mechanism, the estimation of heart rate becomes more accurate.

\section{Experimental Results}

\subsection{Method}

We conducted an experiment on the evaluation of the effectiveness of the proposed method. In the experiment, we used the IEEE Signal Processing Cup 2015 dataset. ${ }^{(13)}$ The dataset included two-channel PPG signal data and 3D acceleration signal data from 12 subjects. In addition, as ground truth data, the accurate heart rate data measured by ECG were included. All signals were recorded for $300 \mathrm{~s}$ at a sampling rate of $125 \mathrm{~Hz}$. During data recording, the subjects walked or ran on a treadmill at the following speeds: $1-2 \mathrm{~km} / \mathrm{h}$ for $30 \mathrm{~s}, 6-8 \mathrm{~km} / \mathrm{h}$ for $60 \mathrm{~s}, 12-15 \mathrm{~km} / \mathrm{h}$ for $60 \mathrm{~s}$, $6-8 \mathrm{~km} / \mathrm{h}$ for $60 \mathrm{~s}, 12-15 \mathrm{~km} / \mathrm{h}$ for $60 \mathrm{~s}$, and $1-2 \mathrm{~km} / \mathrm{h}$ for $30 \mathrm{~s} .^{(13)}$
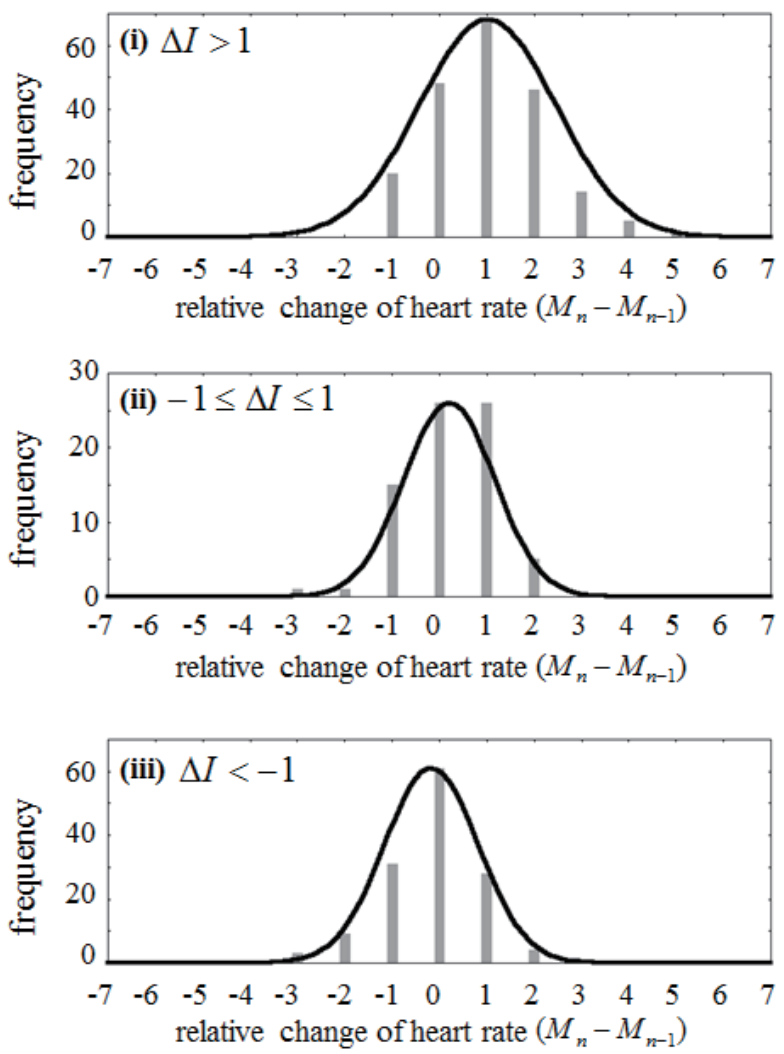

Fig. 3. Histograms of relative change of heart rate at different levels of $\Delta I$
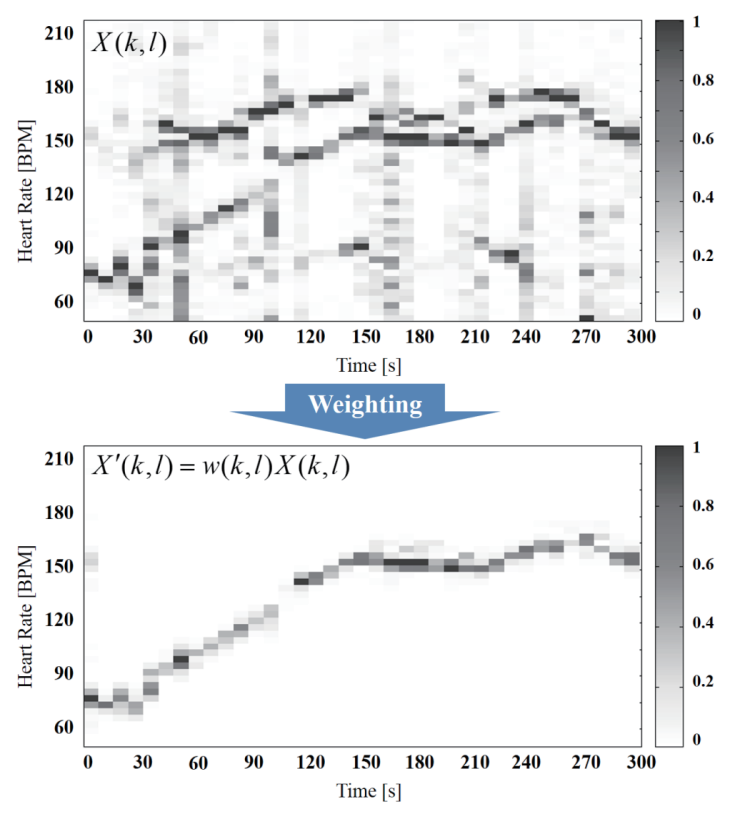

Fig. 4. (Color online) Spectrogram reconstructed by dynamic weighting. 
In the generation of the spectrogram from a PPG signal, the size of the time window in the SFTF was set to 1024 points [ $T=8.192 \mathrm{~s}]$.

In the experiment, we compared the estimated accuracies by the following three methods:

Method 1: simple tracking

The peak-frequency tracking was applied to the original spectrogram $X(n, m)$ without any weighting processing.

Method 2: conventional tracking

The peak-frequency tracking was applied to the weighted spectrogram $X^{\prime}(n, m)$ where the weight matrix $w(n, m)$ was statically defined (i.e., the matrix does not change regardless of exercise intensity). The static weighting approach has been adopted in conventional studies such as Ref. (13). In this method, assuming that exercise intensity was constant (i.e., $\Delta I=0$.), the parameters $\mu(\Delta I)$ and $\sigma(\Delta I)$ in Eq. (5) were set to the constants shown as case (ii) in Table 1.

Method 3: proposed tracking

The peak-frequency tracking was applied to the weighted spectrogram $X^{\prime}(n, m)$, where the weight matrix $w(n, m)$ changed dynamically depending on the level of exercise intensity change, as shown in Table 1.

\subsection{Results}

Table 2 shows the average absolute error (AAE) of the estimated heart rates in the three methods. As shown in this table, by Method 3 (proposed method), the accuracy of heart rate can be greatly improved. These results indicate the effectiveness of the proposed dynamic weighting approach.

Figure 5 shows the spectrogram generated from a PPG signal and the paths of peak-frequency tracking by the three methods. In this figure, the labels VL (very low), L (low), and H (high)

Table 2

Average absolute errors of estimated heart rates in the three methods.

\begin{tabular}{lccc}
\hline & Method 1 & Method 2 & Method 3 \\
\hline AAE (BPM) & 18.76 & 33.86 & 8.98 \\
\hline
\end{tabular}

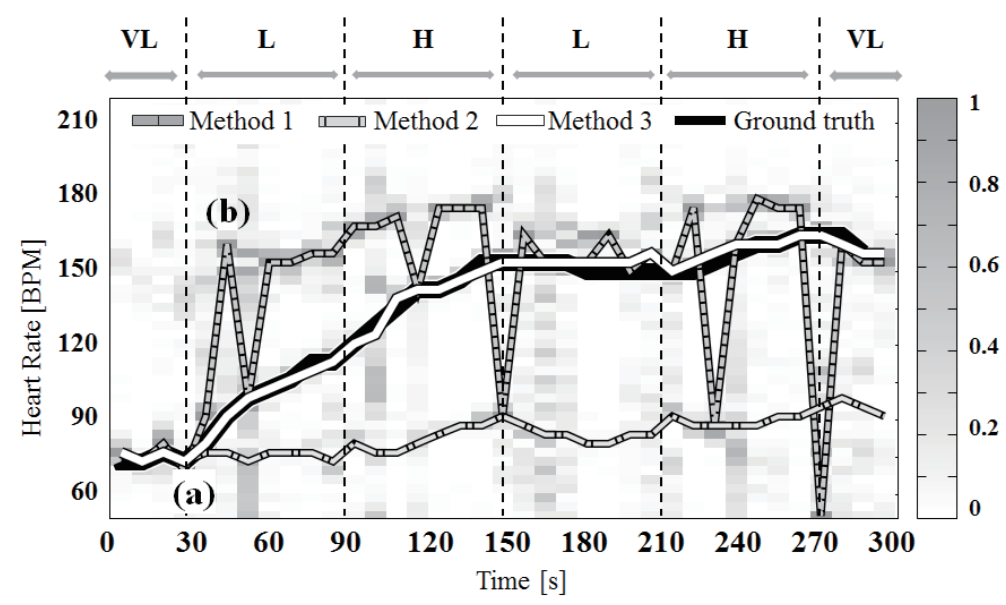

Fig. 5. Peak-tracking results obtained by the three methods. 
attached to each time period represent walking or running speed during the periods. As shown in this figure, the path of Method 3 (proposed method) can accurately capture the correct heart rate in most part. In contrast, the paths of Method 1 and Method 2 are far from the ground truth. During the first $30 \mathrm{~s}$, all the three methods can correctly estimate heart rate. This is because MAs have a negligible effect in this period. The paths, however, diverge after the running speed changes from VL to L [Fig. 5(a)]. By the influence of MA newly added at the timing, the estimated heart rate by Method 1 increases markedly [Fig. 5(b)]. To prevent such a drastic change, Method 2 limits the estimation range of heart rate by weighting the spectrogram. As a result, the heart rate estimated by Method 2 changes gradually. However, the speed of tracking in Method 2 is so low that it cannot capture the rapid change in heart rate when exercise intensity changes. Method 3 (the proposed method) dynamically changes the estimation range depending on the level of exercise intensity change, which is an essential difference from the static weighting adopted in Method 2. From the comparison, we can confirm that, by the dynamic weighting mechanism, the estimation range of heart rate is appropriately controlled depending on the current state of exercise intensity, resulting in a more accurate estimation of heart rate during exercise.

\section{Conclusions}

In this paper, we have proposed a method of accurately estimating heart rate from a PPG signal during exercise considering exercise intensity. From the experimental results, we have confirmed that the proposed method was able to estimate heart rate more accurately than conventional methods.

In this paper, we have mainly focused on the peak-tracking part of the problem of heart-rate estimation from a PPG signal. However, the improvement of the preprocessing part, such as by noise cancellation, is also important for an accurate estimation. Since many techniques for noise cancellation have been studied, we would like to combine these techniques with our method for establishing a more practical heart-rate estimation method.

Heart-rate estimation from PPG signals is a challenging procedure. However, the availability of PPG sensors is promising for smart sensing in the healthcare field. The findings of this study are expected to contribute to future development of smart sensing in this field.

\section{References}

1 J. Allen: Physiol. Meas. 28 (2007) R1.

2 T. Tamura, Y. Maeda, M. Sekine, and M. Yoshida: Electronics 3 (2014) 282.

3 J. Yao and S. Warren: Proc. Int. Conf. Eng. Med. Biol. Soc. 1 (IEEE, 2004) pp. 2153-2156.

4 R. Krishnan, B. Natarajan, and S. Warren: IEEE Trans. Biomed. Eng. 57 (IEEE, 2010) 1867.

5 B. S. Kim and S. K. Yoo: IEEE Trans. Biomed. Eng. 57 (IEEE, 2006) 566.

6 M. Ram, K. V. Madhav, E. H. Krishna, N. R. Komalla, and K. A. Reddy: IEEE Trans. Instrum. Meas. 61 (IEEE, 2012) 1445.

7 R. Yousefi, M. Nourani, S. Ostadabbas, and I. Panahi: IEEE J. Biomed. Health Inf. 18 (IEEE, 2014) 670.

8 H. Fukushima, H. Kawanaka, M. S. Bhuiyan, and K. Oguri: Proc. Int. Conf. Eng. Med. Biol. Soc. (IEEE, 2014) pp. 2901-2904.

9 B. Lee, J. Han, H. J. Baek, J. H. Shin, K. S. Park, and W. J. Yi: Physiol. Meas. 31 (2010) 1585.

10 Y. sheng Yan, C. C. Poon, and Y. Zhang: J. NeuroEng. Rehabilitation 2 (2005) 1.

11 M. Raghuram, K. V. Madhav, E. H. Krishna, and K. A. Reddy: Proc. Int. Conf. Inf. Sci. Signal Process. Appl. (2010) pp. 460-463. 
12 X. Sun, P. Yang, Y. Li, Z. Gao, and Y. T. Zhang: Proc. Int. Conf. Biomed. Health Inf. (IEEE, 2012) pp. 775778.

13 Z. Zhang, Z. Pi, and B. Liu: IEEE Trans. Biomed. Eng. 62 (IEEE, 2015) 522.

14 A. Temko: Proc. Int. Conf. Med. Biol. Soc. (IEEE, 2015) pp. 1500-1503 .

15 Y. Zhang, B. Liu, and Z. Zhang: Biomed. Signal Process. Control 21 (2015) 119.

16 Z. Zhang: IEEE Trans. Biomed Eng. 62 (2015) 1902. 